\title{
CARBAMAZEPINE-INDUCED JUVENILE MYOCLONIC SEIZURES
}

The risk of aggravation of juvenile myoclonic epilepsy (JME) among 40 of 170 consecutive patients who had received carbamazepine (CBZ) or phenytoin (PHT) was determined at the Centre Saint Paul, Marseille, and the Clinique Neurologique, Hopital Pasteur, Nice, France. Seizures were aggravated in $58 \%$ and $15 \%$ benefited. from treatment. Of 28 patients receiving CBZ, $19(68 \%)$ had aggravated symptoms, including myoclonic status in 2 , while $4(14 \%)$ were improved. Of 16 receiving PHT, $6(38 \%)$ were aggravated and $2(12 \%)$ were improved. Half of those benefited had received either valproate or phenobarbital in addition to CBZ or PHT. Vigabatrin with CBZ in one case induced a mixed absence and myoclonic status. (Genton P, Gelisse P, Thomas P, Dravet C. Do carbamazepine and phenytoin aggravate juvenile myoclonic epilepsy? Neurology October (2 of 2) 2000;55:1106-1109). (Reprints: Dr Pierre Genton, Centre Saint Paul, 13258 Marseille 09 France).

COMMENT. Juvenile myoclonic epilepsy usually responds to valproate monotherapy. When misdiagnosed and treated with carbamazepine or phenytoin, myoclonic seizures may be aggravated. The adverse effect of phenytoin appears to be less prominent than that of carbamazepine.

\section{SURGERY FOR EPILEPSY DUE TO CORTICAL MALFORMATION}

Seizure outcome after surgery for focal epilepsy due to malformation of cortical development (MCD), identified by MRI, was evaluated in 35 patients aged 3 months to 47 years (median, 14 years) operated at the Cleveland Clinic Foundation. Outcome classified according to Engel was Class I with no seizures or only auras in 17 (49\%), Class II with rare disabling seizures in 8 (23\%) patients, some improvement in 20\%, and no change in $9 \%$. A seizure-free outcome was more common among patients with complete cf incomplete resection of unilateral MCD (difference NS). MRI evidence of hemimegalencephaly or bilateral MCD was indicative of a poor outcome. (Edwards JC, Wyllie E, Ruggeri PM et al. Seizure outcome after surgery for epilepsy due to malformation of cortical development. Neurology October (2 of 2) 2000;55:1110-1114). (Reprints: Dr Elaine Wyllie, Pediatric Epilepsy Program, Cleveland Clinic Foundation, S51, 9500 Euclid Avenue, Cleveland, OH 44195).

COMMENT. A seizure-free outcome may follow surgical resection in approximately one half of patients with intractable focal epilepsy due to cortical malformation. Complete resection of a unilateral, MRi-defined lesion carries the best prognosis.

\section{ATTENTION DEFICIT DISORDERS}

\section{OUTCOME OF ADHD WITH DEVELOPMENTAL INCOORDINATION}

In a follow-up of 55 of 61 patients at age 22 years who had ADHD with and without comorbid developmental coordination disorder (DCD) at initial workup at 7 years, $58 \%$ of the ADHD/DCD group had a poor outcome compared with $13 \%$ in the comparison group. ADHD complicated by DCD (sometimes called DAMP or MBD) carries an increased risk of poor psychosocial functioning in early adulthood. (Rasmussen P, Gillberg C. Natural outcome of ADHD with developmental coordination disorder at age 22 years: a controlled longitudinal, community-based study. I Am Acad Child Adolesc Psychiatry Nov 2000;39:1424-1431). (Reprints: Dr Rasmussen, Dept Child Psych, Univ of Goteborg, Kungsgatan 12, SE-41119 Goteborg, Sweden). 